\title{
PSEUDO-SASAKIAN MANIFOLDS ENDOWED WITH A CONTACT CONFORMAL CONNECTION
}

\section{VLADISLAV V. GOLDBERG and RADU ROSCA}

\author{
Department of Mathematics \\ N.J. Institute of Technology \\ Newark, N.J. (07l(1)2. U.S.A.
}

(Received January 30, 1985)

\begin{abstract}
Pseudo-Sasakian manifolds $\tilde{N}(U, \xi, \tilde{\sim}, \tilde{g})$ endowed with a contact conforinal connection are defined. It is proved that such manifolds are space forms $\tilde{M}(K), K<0$, and some remarkable propertics of the lie algebra of Infinitesimal transformations of the principal vector field $\tilde{U}$ on $\tilde{M}$ are discussed. Properties of the leaves of a co-isotropic foliation on $\tilde{M}$ and properties of the tangent bundle manifold $T \hat{M}$ having $\tilde{M}$ as a basis are studied.
\end{abstract}

KLY WURDS ANU PHRASES. Witt frame, CICR sulmmifold, relative contact infinitesimal transformation, U-contact concircular pairin:', differential form of Godbillon-Vey, form of E. Cartan, Finslerian jorm, mechinical system, dynamical system, spray, CR product.

1980 SUBJECI' CLASSIFICATION CODES. 5.3C25, 5.:C40, 53B25

1. INTRODUCl'ION.

In the last years many papers have been concerned with Sasakian manifold $\tilde{M}(\phi, \xi, \tilde{n}, \tilde{g})$ and related structures. Recently Rosca [1] has defined pseudo-Sasakian manifolds $\tilde{M}(U, \xi, \tilde{n}, \tilde{g})$ and Goldberg and Rusca [2] have studied CICR submanifolds (i.e. co-isotropic CR submantfolds) of $\hat{M}(U, \zeta, \check{n}, \tilde{g})$.

In the present paper we study $(2 n+1)$-dimenstonal pseudo-Sasakian mantfolds of index $\mathrm{m}+1, \mathrm{~m}>4$, structured by a contri.t conformal (abr. c.c.) connection. It is proved that such manifolds are hyperbolic space forms $\tilde{M}(K), K<0$, and with the c.c. connection (which in fact is a natural seneralization of the connection defined by Rosca [3]) is associated (compare with Rosca [3]) a so denoninated principal vector field Ü.

The paper is organized as follows. In Section 3 we develop some basic results induced by the c.c. connection and some rellirkable properties of the Lie algebra of infinitesimal transformations defined by $i \hat{U}$. It is shown that

(1) $\tilde{U}$ (resp. UUU) is divergence jiec (resp. defines an infinitesimal homothety) on $\hat{M}$ and all connection forms on $\hat{M}$ are integral relations of invariance for UŨ (see Lichnerowicz' [4]):

(ii) $\tilde{U}$ and $U U \tilde{~ d e f i n e ~ a n ~ U-c o n t a c t ~ c o n i n i r c u l a r ~ p a i r i n g ~(i n ~ t h e ~ s e n s e ~ o f ~}$ Rosca [5]) and any contact extension of $\tilde{U}$ is a relative contact 
infinitesimal transformation (in the sense of Rosca [3]) of the canonical 1-form $\tilde{n}$;

(11i) $\tilde{U}$ and $U \tilde{U}$ define both inflinitesimal automorphisms of $(2 q+1)$-forms $\tilde{\beta}_{q}=L \tilde{u}^{q} \quad(q<n)$ where $\tilde{u}$ (resp. L) is the dual form of $\tilde{U}$ (resp. the $(1,1)$-operator taken with respect to the 2 -form $\tilde{\Omega}=d \tilde{n} / 2)$. Accordingly, if $\Sigma_{B}$ is the exterior differentia\} system defined by $\left\{\tilde{\beta}_{q}\right\}, \tilde{U}$ and $U \tilde{U}$ may be considered as isovectors of $\Sigma_{R}$.

Section 4 is concerned with a co-isotrepic foliation $F_{c}$ on $\hat{M}_{\text {. The leaves }}$ $M_{c}$ of $F_{c}$ are CICR submanifolds of $\tilde{M}$ and if $\operatorname{codim} M_{c}=l$, then the form of Godbillon-Vey on $M_{c}$ (see Liclinerowicz $\left.[6]\right)$ is a $(2 \ell+1)$-form $w_{G}$ which is a relative integral invariant of $\mathrm{U}=\left.\tilde{\mathrm{U}}\right|_{\mathrm{M}_{\mathrm{C}}}$.

Further the necessary and sufficient conditions for $M_{c}$ to be foliate is that the isotropic component $U^{+}$of $U$ vanishes. In this case $M_{c}$ is a CR product (see Yano and Kon [7] and Rosca [8]).

Finally using some notions introduced by Yano and Ishihara [9] and also by Klein [10], we consider in Section 5 certilin properties of the tangent bundle manifold Tî̀ having the mantfold $\hat{N}(U, \xi, \hat{n}, \tilde{\varepsilon})$ as a basis.

. It is proved that the complete lifts $\hat{\imath}^{(:}$and $\tilde{u}^{\mathrm{C}}$ of $\tilde{\Omega}$ and $\tilde{u}$ respectively are homogeneous of degree one and that thr firm of E. Cartan $\tilde{\pi}$ on TMे is a Finslerian form. Furthermore, we may associate with $\tilde{\pi}$ a regular mechanical system whose dynamical system is a spraly on $\tilde{M}$.

2. PRELIMINARIES.

Let $(\tilde{N}, \tilde{g})$ be a $(2 m+1)$-dimenstonal connected pseudo-Riemannian mantfold of signature $(m+1, m)$ and suppose that $m>4$.

At each point $\tilde{\mathrm{p}} \in \tilde{\mathrm{M}}$ one has the standard decomposition (see Rosca [1]):

$$
\mathrm{T}_{\mathbf{p}}(\tilde{M})=\operatorname{Hiq}_{\mathbf{p}} \omega \underset{\mathbf{p}}{\tilde{I}}
$$

where $T_{\mathfrak{p}}, H_{p}$, and $T_{\mathfrak{p}}$ are the tangent space, a $(2 \mathrm{~m})$-dimensional neutral vector space, and a time-like line orthogonal to $\|_{p}$, respectively.

Let $\mathbf{s}_{\mathbf{p}}, \mathrm{s}_{\mathbf{p}}^{*} \subset \mathrm{H}_{\mathrm{p}}$ be two self-orthogoncil (abbreviation s.o.) m-distributions which define an involutive automorphism $U$ of square +1 ( $U$ is the para complex operator defined by Libermann [11]). Let $\xi \in T_{\tilde{p}}$ and $\tilde{n} \in \Lambda^{1}(\tilde{N})$ be the pairing which defines a contact structure $\sigma_{c}$ on $\hat{N}$, and $\hat{\gamma}$ be the covartant differentia-

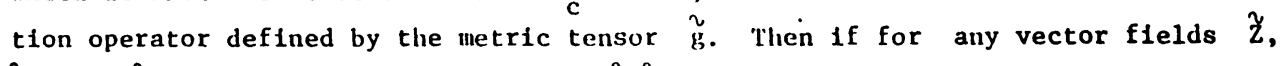
$\tilde{Z}^{\prime}$ on $\tilde{M}$ the structure tensors $(U, \xi, \tilde{n}, \tilde{g})$ satisfy

$$
\begin{cases}u^{2}(\tilde{z})=\tilde{z}-\tilde{n}(\tilde{z}) \xi, & \tilde{g}\left(u \tilde{z}^{2}, u \tilde{z}^{\prime}\right)=-\tilde{g}\left(\tilde{z}, \tilde{z}^{\prime}\right)+\tilde{n}(\tilde{z}) \tilde{n}\left(\tilde{z}^{\prime}\right), \\ \tilde{g}(\tilde{z}, \xi)=\tilde{n}(\tilde{z}), & \tilde{\nabla} \tilde{\eta}, \xi=u \tilde{z}, \\ \tilde{d}\left(\tilde{z}, \tilde{z} \tilde{z}^{\prime}\right)=-2 \tilde{g}\left(u \tilde{z}, \tilde{z}^{\prime}\right), & \tilde{z}^{\prime}(\xi)=1,\end{cases}
$$

the manifold $\tilde{M}(U, \xi, \tilde{n}, \tilde{g}$ ) has been called a pseudo-Sasakian manifold (see Rosca [1]).

In order to study real co-isotropic and isotropic foliations on $\mathcal{M}$ (that is imlroper immersions in $\tilde{M})$, we consider an adapted field of witt fromes: $\tilde{W}=$ $\left\{h_{\Lambda}: A, B, C=0,1, \ldots, 2 m\right\}$. The vectors $h_{A}$ and $h_{a^{*}}\left(a=1, \ldots, m ; a^{*}=a+m\right)$ are null and $h_{0}=\xi$ is the anisotropic vector field of the W-basis $\left\{h_{A}\right\}$. We set

$$
\tilde{S}_{p}=\left\{h_{a}\right\}, \quad \tilde{S}_{p}^{*}=\left\{h_{a} \star\right\}
$$


and as is known, one has

$$
\begin{cases}\tilde{g}\left(h_{a}, h_{b} *\right)=\delta_{a b}, & \tilde{g}\left(\xi, h_{a}\right)=0, \\ \tilde{g}\left(\xi, h_{a} *\right)=0, & \tilde{g}(\xi, \xi)=1\end{cases}
$$

and

$$
U h_{a}=h_{a}, \quad U h_{a} *=-h_{a} *, \quad U \xi=0 .
$$

If $\tilde{W}^{*}=\left\{\hat{\omega}^{\wedge}\right\}$ is the cobasis associated with $\tilde{W}$, we set $\tilde{\omega}^{()}=\tilde{n}$ and the line element $d_{p}^{2}(d \tilde{p}$ is a canonical vector 1 -form and is Independent on any connection on ii) is given by

$$
\tilde{d p}=\stackrel{w}{\omega}_{i \&} h_{\Lambda} \text {. }
$$

It follows from (2.4) that the metric tensor $\tilde{g}$ is:

$$
\tilde{g}=2 \sum_{\mathbf{a}} \tilde{\omega}^{\mathrm{a}} \otimes \tilde{\omega}^{*} \mathbf{a}^{\star}+\tilde{n} \otimes \tilde{n} \text {. }
$$

If $\tilde{\theta}_{B}^{\Lambda}=\tilde{\gamma}_{B C}^{\Lambda} \tilde{\omega}^{C}\left(\tilde{\gamma}_{B C}^{\Lambda} \in C^{\infty}(\tilde{\mu})\right)$ and $\hat{\Theta}_{B}^{\Lambda}$ are the connection forms and the curvature 2-forms on the bundle $\tilde{W}(\hat{N})$ respectively, then the structure equations (E. Cartan) may be written in the indexless form as follows:

$$
\begin{aligned}
& \tilde{V}_{1}=\hat{\theta}>h, \\
& \tilde{d \omega}=-\tilde{\theta} \tilde{\Lambda}, \\
& d \tilde{\theta}=-\tilde{\partial} \hat{A}+\tilde{\Theta} \text {. }
\end{aligned}
$$

Referring to (2.4) and (2.8), one has

and

$$
\left\{\begin{array}{l}
\tilde{\theta}_{b}^{a}+\gamma_{a *}^{*}=0, \quad \tilde{a}_{b}^{*}=0, \quad \gamma_{b}^{*}=0, \\
\tilde{\theta}_{a}^{*}+\gamma_{0}^{*}=0, \quad \gamma_{0}^{* 1}+\gamma_{a}^{*}=0
\end{array}\right.
$$

$$
\tilde{\theta}_{\mathbf{a}}^{0}=\tilde{\omega}^{\mathbf{a}^{*}}, \tilde{0}_{\mathbf{a}}^{*} *=-w^{0} \text {. }
$$

By virtuc of $(2.8),(2.9)$, and $(2.11)$ onc li.1:

and

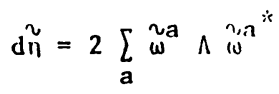

$$
\tilde{\nabla}_{\xi}=\mathrm{Ud}_{\tilde{p}} \Rightarrow\left\langle\tilde{\nabla}_{\tilde{Z}} \xi, \tilde{Z}^{\prime}\right\rangle+\left\langle\tilde{V}_{\tilde{Z}}, \xi, \tilde{Z}\right\rangle=0
$$

where $\tilde{Z}$ and $\tilde{Z}$, are any vector fields on $\hat{H}$.

In the following we agree to call the 2 -form

$$
\tilde{s}=\sum_{a} \stackrel{\sim}{\omega}^{a} \wedge \sim_{\omega}^{\sim} a^{*}
$$

the jumlanmentul 2-form on $\tilde{M}$

Since by (2.11) one has

$$
\tilde{\theta}_{a}^{a}+\tilde{\theta}_{a}^{*}{ }_{a}^{*}=0, \quad \tilde{\omega}_{a}^{a}+\tilde{\Theta}_{a *}^{a^{*}}=0,
$$

we shall call

$$
\tilde{\theta}_{\mathrm{R}}=\sum_{\mathbf{a}} \tilde{\tilde{\theta}}_{\mathbf{a}}^{\mathbf{a}}
$$

and

$$
\tilde{\Theta}_{\mathrm{R}}=\sum_{\mathrm{a}} \Theta_{\mathrm{a}}^{\mathrm{a}}
$$


the Ricci 1-form and the Ricci 2-form respectively (see Rosca [12]). As is known, the form $\tilde{\Theta}_{\mathrm{R}}$ defines the first class of Cherm of $\tilde{M}$.

Using (2.10) and referring to (2.12) and (2.15), one quickly obtains

$$
\tilde{\mathrm{d}}_{\mathrm{R}}=\tilde{\tilde{\theta}} \mathrm{R}-\tilde{s} \text {. }
$$

The above equation proves that the 2-forms $\tilde{\theta}_{\mathrm{R}}$ and $\tilde{\Omega}$ are homologous. Hence the two cocycles $\tilde{\vartheta}_{\mathrm{R}}$ and $\tilde{\Omega}$ belong to the 2-cohomology class $\mathrm{H}^{2}(\tilde{M})$ of $\tilde{M}$.

Let now $F_{c}$ be a coisotropic foliation on $\hat{M}$ and denote by $M_{c}$ a maximal integral manifold (leave) of $\Gamma_{c}$. It has been shown by Goldberg and Rosca [2] that $M_{c}$ is a contact $C R$ submanifold of $\ddot{M}$, thit is there exists a differentiable distribution $D: p \rightarrow D_{p} \subset T_{p}\left(M_{c}\right), p \in M_{c}$ (one denotes the Induced elements on $M_{c}$ by suppressing $\sim$ ) satisfying:

(i) $D$ is invariant i.e. $U \mathrm{UD}_{\mathrm{p}} \subseteq \mathrm{D}_{\mathrm{p}}$, and

(ii) the complementary orthogonal distribution $D^{\perp}: p \rightarrow D_{p}^{\perp} \subset T_{p}\left(M_{c}\right)$ is antiinvuriant i.c. UU ${ }_{\mathrm{p}}^{\perp} \subseteq \mathrm{T}_{\mathrm{p}}^{\perp}\left(\mathrm{M}_{\mathrm{c}}\right)$.

The distribution $\mathrm{D}$ (resp. $D^{L}$ ) is called the horizontal (resp. vertical) distribution. Such type of CR submanifolds is called CICR submanifolds (see Goldberg and Rosca [2]).

3. PSEUDU-SASAKIAN MANIFOLDS ENDUWED WITH $\Lambda$ CUNTACT CONFORMAL CONNECTION.

As a natural generalization of the definttion given by Rosca [3], we assume that the structure equations (2.9) are written in the form

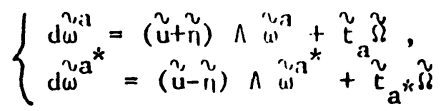

where $\tilde{\Omega}=\tilde{d} \tilde{n} / 2, \tilde{t}_{a}, \tilde{t}_{a^{*}} \in C^{\infty}(N)$, and $u \in \Lambda^{1}(\tilde{N})$ is a closed 1-form. Note that $\tilde{t}_{a}$ and $\tilde{t}_{a}{ }^{*}$ are the components of a vector field

$$
\tilde{U}=\sum_{a}\left(\tilde{t}_{i} h_{a}+\tilde{t}_{a} * h_{a}\right)
$$

of constant length.

We shall say (see Rosca [3]) that in this case the pseudo-Sasakian manifold $\tilde{M}$ is endowed with a contact conformal (abr. c.c.) connection. We also agree to call $\tilde{U}$ the principal vector field associated with this connection.

Since $\tilde{g}(\tilde{U}, \tilde{U})=$ const, we may write by (3.2) that

$$
\sum_{a}^{2} a_{a}^{*} \tilde{t} a^{*}=c, c=\text { const. }
$$

Taking exterior differentials of (3.1), we get

$$
\left\{\begin{array}{l}
d \tilde{t}_{a}=(\tilde{u}+\tilde{i}) \tilde{t}_{a}-2 \tilde{w}^{\prime a}, \\
d \tilde{t}_{a^{*}}=(\tilde{u}-\tilde{\eta}) \tilde{t}_{a^{*}}-2 \tilde{\omega}^{*}
\end{array} .\right.
$$

Denote by $\Sigma$ the exterior differential system defined by equations (3.1) and (3.4) and by $I$ the ideal corresponding to $\Sigma$. The exterior differentiation of (3.4) where $\tilde{\omega}^{a}$ and $\tilde{\omega}^{a^{*}}$ satisfy $(3.1), \tilde{\Omega}=\mathrm{d} \tilde{n} / 2, \mathrm{du}^{*}=0$, leads to the identity. Because of this, $\mathrm{dI} \subset \mathrm{I}$, that is $\Sigma$ is a closed system. It follows from this that the system $\Sigma$ defining the pseudo-Sasakian manifold $\tilde{M}$ endowed with a c.c. connection is completely interrable and tis solution depends on $2 \mathrm{~m}$ constants (the number of equations in $(3.4)$ ). 
From (3.4) and (3.3) we also obtain

$$
\tilde{c u}=\sum_{a}\left(t a^{* \omega^{a}}-t_{a} \omega^{a^{*}}\right)
$$

and $\tilde{u}(\tilde{U})=0$ which shows that $\tilde{u}^{\mathbf{a}}$ is an integral relation of invariance for $\tilde{U}$ (see Liclinerowicz [4]). In the following we agree to call $\tilde{u}$ the principal Pfaffian associated with the c.c. connection.

Consider now the 1-form

$$
\tilde{v}=\sum_{a}\left(\tilde{t} \tilde{a}^{\sim a^{*}}+\tilde{t}+\tilde{a}^{* a}\right)
$$

Taking the exterior differential of $\tilde{v}$, one finds with the help of (3.1) and (3.4) that $c=2$. In this case we deduce

$$
d \tilde{v}=2 \tilde{u} \wedge \tilde{v}
$$

and this equation asserts that $\tilde{v}$ is extorior recurrent (see Datta [13] with 2ü as the recurrence $1-\int 0 r m$.

By (2.4) and (2.5) one easily finds

$$
\tilde{u}(U \tilde{U})=\tilde{v}(\tilde{U})=\tilde{g}(\tilde{U}, \hat{U})=\tilde{k}(1 \tilde{U}, U \tilde{U})=2\left[\tilde{t} \tilde{t}_{a} \tilde{t}^{*} .\right.
$$

Hence if $b: \mathrm{T}(\tilde{M}) \rightarrow \mathrm{T}^{*}(\tilde{M})$ is the musicul iromorpliism with respect to $g^{*}$ (see Poor [14]), we may write: $\tilde{u}=b(U \tilde{U}), \tilde{v}=b$ (î). Since $\tilde{u}$ is closed, it follows from (3.7) that the manifold $\tilde{M}$ under consideration is follated by 2-codimensional submanifolds orthogonal to $\tilde{U}$ and $U \tilde{U}$.

Next if $\mu: \tilde{Z} \rightarrow 1 \tilde{Z} \tilde{\Omega}, T(\tilde{M})+T^{*}(\tilde{N})$ is the bundle 1somorphism defined by $\tilde{\Omega}=\mathrm{d} \tilde{n} / 2$, one readily finds

$$
\mu(\tilde{u})=2 \hat{i} .
$$

In the following we agree to call the presympletic form $\tilde{\Omega}(\mathrm{dim} \operatorname{ker}(\tilde{\Omega}) \neq 0)$ the fundamental 2-form on $\hat{\mu}$.

Let now $\tilde{U}_{f}=\tilde{U}+\tilde{f} \xi \quad\left(\tilde{Y} \in C^{\infty}(\tilde{M})\right)$ be a contact extension of $\tilde{U}$ and $\mathscr{L}_{\tilde{U}_{f}}$ the Lie derivative with respect to $\tilde{U}_{f}$. Then by (3.9) one quickly finds d $\mathscr{U}_{f} \tilde{n}=0$. Therefore according to the definttion given by Rosca [3], we may say that $\tilde{U}_{f}$ is a relative contact infinitesimal transformation of $\tilde{n}$.

Denote now by $\tilde{\sigma}_{S}$ (resp. $\tilde{\sigma}_{S^{*}}$ ) the simple unit form which corresponds to $\tilde{S}_{p}$ (resp. $\underset{\mathrm{p}}{\mathbf{\mathrm { f }}}$ ). . One has

$$
\left\{\begin{array}{l}
\tilde{\sigma}_{s}=\tilde{\omega}^{1} \wedge \ldots \wedge \omega_{\omega}^{n, m}, \\
\tilde{\sigma}_{s}^{*}=\tilde{\omega}^{1^{*}} \wedge \ldots \wedge \omega_{\omega}^{\sim m}
\end{array},\right.
$$

and by (3.1) the exterior differentials of (3.10) are

$$
\left\{\begin{array}{l}
\tilde{d}_{S}=[m(\tilde{u}+\tilde{n})-\tilde{v}] \wedge \tilde{\sigma}_{S} \\
d \tilde{\sigma}_{S}^{*}=\left[m(\tilde{u}-\tilde{n})+\tilde{v}^{\tilde{v}}\right] \wedge \tilde{\sigma}_{S}^{*} .
\end{array},\right.
$$

Since $\tilde{\sigma}_{S}$ and $\tilde{\sigma}_{S}$ are both exterior recurrent, it follows from a well-known property that both co-isotropic distributions $\tilde{S}+\{\xi\}$ and $\tilde{S}^{*}+\{\xi\}$ are involutive (orth. $(\tilde{S}+\{\xi\})=\tilde{S}$; orth. $\left.\left(\tilde{S}^{*}+\left\{\xi_{3}\right\}\right)=S^{*}\right)$. It is worth to emphasize that this property is true for any pseudo-Sasakian manifold. 
Now with the help of (3.1), one finds that the connection forms are given by

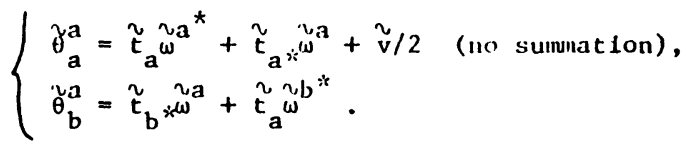

By (3.12) and (3.6) one finds

$$
\tilde{\theta}_{R}=(n+2) \hat{v} / 2
$$

and (3.7) shows that $\tilde{\theta}_{\mathrm{R}}$ is exterior recurrent.

Comfing back to relations (3.12), one reildily finds

$$
\gamma_{a}^{a}(u \hat{u})=0, \quad \tilde{0}_{b}^{a}(u \hat{i})=0 .
$$

Therefore we may say that all connection forms of the pseudo-Sasakian manifold $\tilde{M}$ under consideration are integral relations of invariance for the vector field UÜ. Denote now by $\tilde{\tau}$ the volume element of $\tilde{M}$. One may take a local orientation such that

$$
\tilde{\tau}=\tilde{\sigma}_{S} \Lambda \tilde{\sigma}_{S} * \Lambda \tilde{n}
$$

and denote by $*: \Lambda \mathrm{q}^{*} \tilde{M} \rightarrow \Lambda^{2 \mathrm{n}+1-\mathrm{q}_{\mathrm{T}} * \tilde{M}}$ the star operator determined by $\tilde{\tau}$. If, 1 ike usual1y, $X \tilde{M}$ means the vector space of sections over $T \tilde{M}$, then, as is known, for any vector field $\tilde{Z} \in \mathcal{H}$ one has

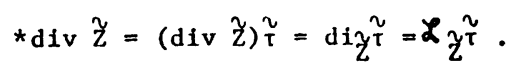

Making use of $(3.4),(3.11),(3.16)$, and the fact that

$$
\tilde{U}=\sum_{a}\left(\tilde{t}_{a} h_{a}+\tilde{t}_{a} * h_{a}\right),
$$

one finds after some calculations:

$$
\operatorname{div} \tilde{U}=0, \operatorname{div}(\tilde{U})=2 \sum_{a} \tilde{t_{a}} \tilde{t}_{a}^{*}=4 .
$$

Hence $\tilde{U}$ is divergence free and $\tilde{U}$ is an infinitesimal homothety on $\hat{M}$.

Now if $\tilde{Z}=\tilde{Z}^{A_{h}}{ }_{A^{\prime}} \tilde{Z}^{\prime}=\left(\tilde{Z}^{\prime}\right){ }^{A_{h}} \Lambda \varepsilon \tilde{M}$ are any vector fields, then, as 1s known (see Poor [14]), one has

$$
\tilde{\nabla}_{\tilde{Z}}, \tilde{Z}=\left(\mathscr{d}_{\tilde{Z}}, \tilde{z}^{A}\right) h_{\Lambda}+\tilde{z}^{A}\left(\tilde{\nabla}_{\tilde{Z}}, h_{A}\right) \cdot
$$

Therefore, by $(2.3),(3.4)$, and $(3.12)$ we get

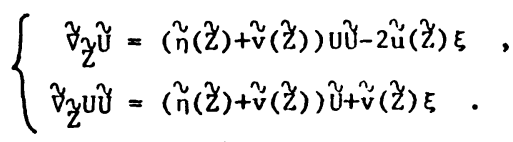

We also note that since $b(U U \tilde{)})=\tilde{u}$ is a closed form, we may say (see Poor [14]) that $\forall$ uU is self-adjoint.

According to the definition given by Rosca [5] and Rosca and Verstraelen [15], the formulae (3.19) show that the vector field $\tilde{U}$ defines a $U$-contact concircular pairing.

Denote by $D_{U}$ the 3-distribution defined by $\{\tilde{U}, U \tilde{U}, \xi\}$. By (2.2), (3.5), and (3.6) one readily finds from (3.19) that

$$
[\tilde{U}, \xi]=0, \quad[u \tilde{U}, \xi]=0 \text {. }
$$

Hence both vector flelds $\tilde{U}$ and $U \tilde{U}$ commute with $\xi$ and by (3.19) and (3.20) we see that $D_{U}$ defines a 3-foliation on $\hat{M}$. 
It is worth now to make the following considerations.

Let $\tilde{Z} \in \mathcal{X} \tilde{M}$ be any vector field on $\tilde{M}$. Then one has the general Bochner Sormula (see Poor [14]) on $\tilde{M}$ :

$$
2<\operatorname{tr} \tilde{\nabla}^{2} \tilde{z}, \tilde{Z}>+2\|\tilde{v} \tilde{z}\|^{2}+\check{x}\|\tilde{Z}\|^{2}=0
$$

where $\tilde{\Delta}=\operatorname{do} \delta+\delta$ od is the Laplace-Rnltruni cperater (or Laplacian) on $\Lambda \mathrm{r} * \tilde{M}$, and the trace (abr. $t r)$ is calculated with respect to the metric tensor $\tilde{g}$ of $\tilde{M}$. Applying formula (3.21) to the princip.l vector field $\tilde{U}$ and taking into account (2.7), one has

$$
\operatorname{tr} \tilde{\nabla}^{2} \tilde{U}=\sum_{a} \tilde{\nabla}_{h_{a}}\left(\tilde{\nabla}_{h_{a^{*}}} \tilde{u}\right)+\sum_{a} \ddot{v}_{h_{a^{*}}}\left(\tilde{\nabla}_{h_{a}} \tilde{u}\right)+\tilde{\nabla}_{\xi}\left(\tilde{\nabla}_{\xi} \tilde{u}\right)
$$

and

$$
\|\tilde{\nabla} \hat{u}\|^{2}=2 \sum_{a}\left\langle\tilde{\nabla}_{h_{a}} \tilde{U}, \tilde{\nabla}_{h_{a^{*}}} \tilde{u}\right\rangle+\left\langle\tilde{v}_{\xi} \tilde{u}, \tilde{\nabla}_{\xi} \tilde{U}\right\rangle \text {. }
$$

Now by $(2.14),(3.4),(3.5),(3.16)$, and (3.19) one finds

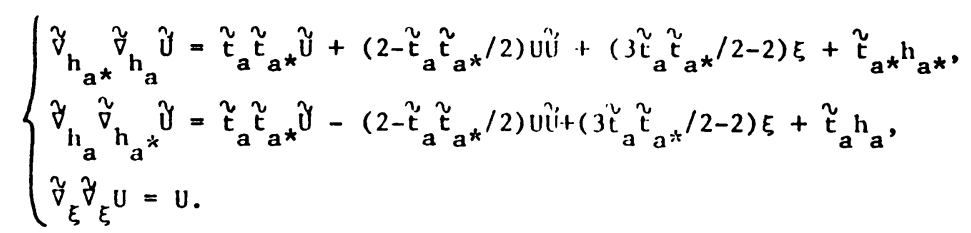

Since we have found $\sum_{\tilde{t}_{a}} \tilde{t}_{a *}=2$, we derlve from $(3.22),(3.23),(3.24)$, and (3.21) that $\tilde{U}$ satisfies $(3.21)$ and this equation is consistent with $\|\tilde{U}\|^{2}=4$.

Let $\mathrm{L}$ be the operator of type $(1,1)$ defined by the fundamental 2-form $\tilde{\Omega}$. Denote then by $\tilde{\xi}_{q}=L^{q} \tilde{u}=\tilde{u} \Lambda(\Lambda \tilde{\Omega})^{q} \in \Lambda^{2 q+1} \tilde{M}$. Since $\tilde{u}$ and $\tilde{\Omega}$ are both closed, one finds by (3.9) and making use of the properties of the Lie derivative $\mathscr{L}=1 \circ d+d \circ 1$ that

$$
\mathcal{L} \underset{\tilde{U}^{\mathrm{B}}}{\mathrm{r}}=0 \text {. }
$$

Hence $\tilde{U}$ is an infinitesimal automorphism of a11 $(2 q+1)$-forms $\tilde{B}_{q} \quad(q<m)$.

On the other hand, since $\tilde{g}(\tilde{U}, \tilde{U})=$ const, we may say in simllar manner as in the case of a Sasakian manifold that $\tilde{U}$ defines with UŨ an U-section.

Like usually denote by

$$
R\left(\tilde{Z}, \tilde{Z}^{\prime}\right)=\left[\tilde{\nabla} \tilde{Z}, \tilde{\nabla}_{\tilde{Z}^{\prime}}\right]-\tilde{\nabla}_{\left[\tilde{Z}, \tilde{Z}^{\prime}\right]}, \tilde{Z}, \tilde{Z}^{\prime} \in \mathcal{X} \tilde{M}
$$

the curvature operator. Then, as is known, the sectional curvature $\mathrm{K}(\tilde{U}, \mathrm{U} \tilde{)})$ defined by $\tilde{U}$ and $U \tilde{U}$ is given by

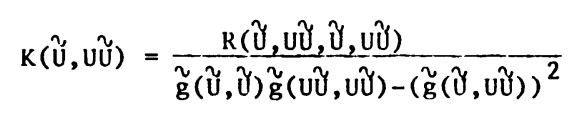

where

$$
R(\tilde{U}, U \tilde{U}, \tilde{U}, U \tilde{U})=\tilde{g}(R(\tilde{U}, U \tilde{U}) \cup U ̛, \tilde{U}) .
$$

Making use of $(3.5),(3.6)$, and $(3.19)$, one finds

$$
[\tilde{U}, \tilde{U}]=4(\hat{U}+2 \xi)
$$

and

$$
R(\tilde{U}, U \tilde{U}) U \tilde{U}=4(5 \tilde{U}+8 \xi)
$$


Hence by (3.27) and (3.28) one gets $k(i, u(i))=-\frac{1}{5}$. Now referring to (2.10) and (3.12) one finds after some calculations

$$
\begin{aligned}
& \tilde{\Theta}_{a}^{a}=\tilde{v}_{S} \Lambda \tilde{v}_{S^{*}}+\tilde{v}_{S} \Lambda \tilde{t}_{a^{(j)}}^{v^{*}}-\tilde{v}_{S^{*}} \Lambda \tilde{t}_{a^{*}}{ }^{(\omega)}
\end{aligned}
$$

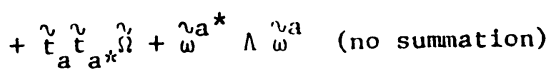

where we have set

$$
\left\{\begin{array}{l}
\tilde{v}_{s}=\sum \tilde{t}_{a^{*}} \tilde{w}^{a} \varepsilon \Lambda^{1} \tilde{s}, \\
\tilde{v}_{S^{*}}=\sum \tilde{\mathrm{t}}_{\mathrm{a}^{w}} \tilde{a}^{*} \varepsilon \Lambda^{1} \tilde{\mathrm{s}}^{*} .
\end{array}\right.
$$

As is known (see Libermann [11]), the components of the Ricci tensor are given by $\tilde{\Theta}_{a}^{a}=\tilde{R}_{b c} \star^{w b} \wedge \tilde{\omega}^{c *}\left(\tilde{\mathcal{G}}_{a}^{a}+\tilde{\mathcal{G}}_{a}^{a^{*}}=0\right)$. Because of this, we get from (3.31) that

$$
\left\{\begin{array}{l}
\tilde{\mathrm{R}}_{\mathrm{bc}} *=\tilde{\mathrm{t}}_{\mathrm{b}^{*}} \tilde{\mathrm{t}}_{\mathrm{c}}, \\
\tilde{\mathrm{R}}_{\mathrm{aa}} *=2 \tilde{t}_{\mathrm{a}} \tilde{\mathrm{t}}_{\mathrm{a}} *-1 .
\end{array} .\right.
$$

It follows from (3.33) that the components of the Ricci tensor are disjoint (see Rosca [16]). In addition, since the scalar curvature $\tilde{C}_{s}$ is the trace of the Ricci tensor with respect to $\tilde{g}$, one finds by (2.7) and (3.3) that $\tilde{\mathrm{C}}_{\mathbf{s}}=4-\mathrm{m}(\mathrm{m}>4)$. Therefore we conclude that the pseudo-Sasakian mantfold $\tilde{M}$ under consideration is a space form $\tilde{M}(4-\mathrm{m})$ of hyperbolic type.

THEOREM 1. Let $\tilde{M}(U, \xi, \tilde{n}, \tilde{g})$ be a pseudo-Sasakian manifold endowed with a c.c. connection and let $\tilde{U}$ (resp. $\tilde{\Omega}=\mathrm{d} \tilde{n} / 2$ ) be the principal vector field associated with this connection (resp. the fundamental 2-form on $\tilde{M}$ ). One has the following properties:

(1) $\tilde{U}$ is divergence free, and Uư defines an infinitesimal homothety on $\tilde{M}$;

(11) all the connection forms on $M$ are Integral relations of invariance for uÚ;

(111) Ũ and UŨ define an U-contact concircular pairing, and $\{\tilde{U}, U \tilde{U}, \xi\}$ defines a 3-follation on $\tilde{M}$;

(iv) any contact extension $\tilde{U}_{f}=\hat{U}+\tilde{f} \xi$ of $\tilde{U}$ is a relative contact infinttesimal transformation of $\tilde{n}$;

(v) $\tilde{U}$ and $U \tilde{U}$ define both an Anfinitesimal automorphism of all (2q+1)-forms $\tilde{B}_{q}=L^{q} \tilde{u}$ where $\tilde{u}$ is the dual form of $U \tilde{U}(q<m)$;

(vi) the Ricci 1-form of $\hat{M}$ is exterior recurrent, and the Ricci tensor is disjoint;

(vii) $\tilde{M}$ is a space-form of hyperbolic type;

(vi11) any such submanifold $\tilde{M}$ is defined by a completely integrable system of differential equations whose solution depends on $2 \mathrm{~m}$ arbitrary constants.

4. CO-ISOTROPIC FOLIATION ON $\tilde{M}(U, \xi, \tilde{n}, \tilde{g})$.

We shall consider on $M$ the following three distributions:

a) An invariant distribution $\mathrm{D}^{\boldsymbol{T}}$ (i.e. UD $\subseteq \mathrm{D}^{\boldsymbol{T}}$ ) of dimension $2(\mathrm{~m}-\mathrm{l})+1$ defined by $D^{T}=\left\{h_{1}, h_{1 *}, \xi ; 1=1, \ldots, m-l ; i^{*}=1+m\right\}$.

b) An isotropic distribution $D^{\perp}$ (i.e. $D^{\perp} \subseteq$ orth $D^{\perp}$ ) of dimension $l$ defined by $D^{\perp}=\left\{h_{r} ; r=m-\ell+1, \ldots, m\right\}$. 
c) $\Lambda$ transversal distribution $v_{t}=l_{S} *\left(v^{\top} \oplus \mathrm{D}^{\perp}\right)\left(r \mathrm{~s}^{\star}\right.$ of dimension $\ell$ defined by $v_{t}=\left\{h_{r^{*}} ; r^{*}=2 m-\ell+1, \ldots, 2 m\right\}$.

These three distributions have no comnon direction and they define on $\tilde{M}$ a $f$-structur'e of rank $2 l$ (see Sinha [17]).

Accordingly we shall split the principal vector field $\tilde{U}$ as follows:

$$
\tilde{u}=\tilde{u}^{\top} \oplus \hat{u}^{\prime} \oplus \hat{u}_{t}
$$

where $\tilde{U}^{\top} \in D^{\top}, \tilde{U}^{\perp} \in D^{\perp}, \tilde{U}_{t} \in D_{t}$.

Denote now by

$$
\tilde{\Psi}=\omega_{\omega}^{n 2 m-\ell+1} \wedge \ldots \varkappa_{\omega}^{2 m}
$$

the simple unit form which corresponds to $D_{t}$. Because $D_{t}$ is orientable, $\tilde{\psi}$ is a well-defined global form. Since $\dot{\psi}$ annihilates $D^{\boldsymbol{T}} \oplus D^{\perp}$, the necessary and sufficient condition for $\mathrm{D}^{\boldsymbol{T}} \oplus \mathrm{D}^{\perp}$ to be a co-isotropic foliation $F_{\mathrm{c}}$ is that $\tilde{\psi}$ be exterior recurrent (see Lichnerowicz [18] and Yano and Kon [7]).

Hence one must write $d \tilde{\psi}=\tilde{\gamma} \Lambda \tilde{\psi}$ and if $H^{1}\left(F_{c}, R\right)$ represent the 1-cohomology class of $F_{c}$, then the recurrence 1 -form $\tilde{r}$ defines an element of $H^{1}\left(F_{c}, R\right)$ (see Iflchnerowlcz [6]). In the case under discussion one finds (compare with Yano and Kon [7]) that the necessary and sufficient condition for $\mathcal{M}$ to receive a co-isotroplc follation $F_{c}=D^{\top} \oplus D^{\perp}$ is that the component $\tilde{U}_{t}$ of $\tilde{U}$ vanishes. In this case the recurrence 1 -form $\tilde{\gamma}$ of $\tilde{\psi}$ is given by

$$
\tilde{r}=\ell(\tilde{u}-\tilde{r}) \text {. }
$$

Denote by $M_{c}$ a $(2 m-\ell+1)$-dimensional leaf of $F_{c}$ and supress $\sim$ for the induced elements on $M_{c}$.

According to the considerations of Section 1 , it follows that $M_{c}$ is a CICR submanifold. By definition we have du $=0$. Because of this and $(3.1)$, the exterior differentiation of (4.3) gives

$$
\mathrm{d} \gamma=-2 \ell \Omega \text {. }
$$

Equation (4.4) shows that the restriction $\Omega=\left.\tilde{\Omega}\right|_{M_{c}}$ is an exact form.

On the other hand, the form of Godbillon-Vey (see Lichnerowicz [6]) on $M_{c}$ is the $(2 \ell+1)$-form $w_{G} \varepsilon \Lambda^{2 \ell+1}\left(M_{c}\right)$ given by

$$
w_{G}=\gamma \wedge(\Lambda \mathrm{d} \gamma)^{\ell} \text {. }
$$

One knows (see Lichnerowicz [18]) that the class of cohomology of ${ }_{G}$ which is an element of $\mathrm{H}^{2 \ell+1}\left(M_{c} ; R\right)$ is an invariant of the follation. Using the same notation as in section 3 and applying $(4.4)$, we may write

$$
w_{G}=c\left(L^{\ell} u-L^{\ell} n\right)-c\left(B_{\ell}-L^{\ell} n\right)
$$

where we have set $c=-2^{\ell} \ell^{\ell+1}$.

Thus it follows from (3.22) that

$$
\mathscr{L}_{U}{ }^{w_{G}}=-c \mathcal{L}_{U}\left(L^{\ell} n\right) \text {. }
$$

By means of (2.13) and (3.9) one has

$$
d\left(L^{\ell} n\right)=2(\Lambda \Omega)^{\ell+1}
$$


and

$$
d \mathbf{d}_{U}\left(L^{\ell} n\right)=4 \ell u(\Lambda \Omega)^{\ell} .
$$

Therefore we get

$$
\mathscr{L}_{U}\left(L^{\ell} n\right)=-11 \wedge(\Lambda \Omega 2)^{\ell}=-\beta_{\ell}
$$

and finally

$$
\mathcal{L}_{U}{ }^{w}\left(:=c R_{l} .\right.
$$

Since $B_{\ell}$ is closed, the above equation gives $d \mathscr{L}_{U} w_{G}=0$ and allows us to say that $w_{G}$ is a relative integral invariant of $\mathrm{u}$.

Further since the submanifold $M_{c}$ is co-isotropic, it follows from this that the normal bundle $T^{\perp} M_{c}$ of $M_{c}$ coincides with $D^{\perp}$.

Since $N_{c}$ is defined by ${ }_{\omega}{ }^{\star}=0, r^{*}=2 m-\ell+1, \ldots, 2 m$, we derive from $(2.8)$ and (3.12) that the covariant derivatives $V_{h}$ of the null normal sections $h_{r}$ satisfy

$$
\nabla h_{r}=\frac{v}{2} \otimes h_{r} .
$$

Since $h_{r}$ are null vector fields, equation (4.12) shows that $h_{r}$ are geodesic direptions. Hence according to the definition of Rosca [19], one may say that $D^{\perp}$ has the geodesic property.

Further if $X$ and $Y$ are any vector fields of $D^{\perp}$, one has $\nabla_{Y} X \in D^{\perp}$. Thus according to a known definition, the distribution $\mathrm{D}^{\perp}$ is autoparallel.

Setting $\ell_{r}=-\left\langle\mathrm{dp}, \nabla h_{\mathbf{r}}\right\rangle$ for the second fundomental quadratic forms associated with the inproper inmersion $x: M_{c} \rightarrow M \quad\left(l_{r}\right.$ is a field of symmetric covariant tensors of order 2 on $M_{c}$ ), we derive by a simple argument that all ${ }_{r}$ vantsh. Therefore according to a well-known defintion, we agree to say that the improper immersion $x: M_{c}+\hat{M}$ is improper totally orodesic.

It was proved by Goldberg and Rosca [2] that the distribution $D^{\perp}$ is always involutive. If $M^{+}$are the leaves of 1$)^{\perp}$, then in a similar manner as for $M_{c}$ one easily finds that the improper immersion $x: M^{+}+\tilde{M}$ is improper totally geodesic. Since $\mathrm{x}: \mathrm{M}^{\boldsymbol{T}} \rightarrow \tilde{M}$ is a proper immersion, it is totally geodesic.

Next as it was proved (Goldberg and Rosca [2]) the necessary and sufficient condition for the manifold $M_{c}$ to be fuliate is that the simple unit form $\psi$ which corresponds to $D^{\perp}$ be exterior recurrent.

Since obviously one has $\phi=\omega^{m-\ell+1} \Lambda \ldots \Lambda \omega^{m}$, then by (3.1) one finds that the property of exterior recurrency for $\phi$ ts equivalent to the condition $U^{\downarrow}=0$.

Since by definition in this case $D^{\top}$ is involutive, let us denote by $M^{\top}$ a $(2(m-l)+1)$-dimensional leaf of $D^{\top}$. Because $M_{c}$ is a CICR submantfold, $M^{\top}$ is as is known an Invariant subinanifold of $\tilde{M}$, and this implies (see Rosca [1]) that $M^{\boldsymbol{T}}$ is minimal.

Coming back to the case under discussion, using (2.8), (3.12) and the fact that on $M$ one has $U_{t}=0, U^{\dagger}=0$, we can show by means of a simple calculation that $M^{\top}$ is also totally geodesic.

Hence $M_{c}$ is foliated by two families of orthogonal totally geodestc submanifolds $\mathrm{N}^{\mathrm{C}}$ and $\mathrm{M}^{\mathrm{T}}$. 
On the other hand, let $X \in X M_{c}$ be any vector field on $M_{c}$. According to Rosca [1], one has $\mathrm{UX}=\mathrm{PX}+\mathrm{FX}$ where $\mathrm{PX}$ (resp. FX) is the tangential (resp. the normal) component of UX. By virtue of the total geodesicity of $M^{\top}$, one easily finds that $\nabla P X \in M^{T}$.

Therefore the tangential component $\mathrm{pX}$ of $\mathrm{X}$ is parallel. According to Yano and Kon [7], it follows from this that $M_{c}$ is a CR product 1.e. $M_{c}=M^{+} \times M^{\top}$.

Since $M_{c}$ is connected, this property can be checked by de Rham decomposition theorem.

It is worth to note that this situation is quite similar to that of co1sotropic CR submantfolds of a para Kachlerian manifold structured by a geodesic comection (Rosca [20]).

THEOREM 2. Let $M$ be a pseudo-Sasakian manifold structured by a c.c. connection and let $\tilde{U}$ be the principal vector field associated with this connection. Then the necessary and sufficient condition for $\tilde{M}$ to receive a co-isotropic follation $\Gamma_{c}$ is that the transversal component $\tilde{U}_{t}$ of $\tilde{U}$ vanishes. In this calse the leaves $M_{c}$ of $F_{c}$ are $C I C R$ submanifolds of $M_{c}$, and if $\operatorname{codim} M_{c}=l$, the form of Godbilion-Vey on $M_{c}$ is a $(2 \ell+1)$-form ${ }_{\hat{G}} \hat{G}$ which is a relative integral invariant of $U=\left.\tilde{U}\right|_{M_{C}}$.

In addition, one has the following properties:

(1) the improper immersion $x: M_{c} \rightarrow \tilde{M}$ is improper totally geodesic;

(1i) $\mathrm{N}_{c}$. Is foliated by anti-1nvariant subinanifolds $\mathrm{M}^{+}$which are Improper totally geodesic and have the geodesic property.

Further the necessary and sufficient condition for $M_{c}$ to be follate is that the vertical (or isotropic) component $U^{\perp}$ of $U=\left.\tilde{U}\right|_{M_{C}}$ vanishes. In this case $M_{c}$ is a CR product.

5. TANGENT BUNDLE MANIFOLD TḾ.

Let TMI be the tangent bundle manifinld having the pseudo-Sasakian mantfold discussed in Section 3 as a basis.

Denote by $\tilde{V}_{\mathrm{L}}\left(\mathrm{v}^{\mathrm{A}}\right)$ the canonical vector' sield (or the vector field of Liouvilze) on TM̃. Accordingly we may consider the set $B^{*}=\left\{\tilde{\omega}^{\sim} A, d v^{\sim}\right\}$ as an adapted cobasis on Tĩ. Following codbillon [21], we shall designate by $d_{v}$ and ${ }_{v}$ the vertical differentiation and the vertical derivation operators, respectively taken with respect to $B^{*}\left(d_{v}\right.$ is an antiderivation of degree 1 of $\Lambda$ TM and $1_{v}$ is a derivative of degree 0 of $\left.\Lambda \mathrm{TM}\right)$.

Let $T_{s}^{r} \mathbb{M}$ be the set of all tensor fields of type $(r, s)$ on $\mathcal{M}$. In general the vertical and complete lifts are linear mappings of $T_{s}^{r} \tilde{M}$ into $T_{s}^{r} T$, and for complete lifts one has:

$$
\left(\mathrm{T} \otimes \mathrm{T}_{2}\right)^{\mathrm{C}}=\mathrm{T}_{1}^{\mathrm{V}} \rightarrow \mathrm{T}_{2}^{\mathrm{C}}+\mathrm{T}_{1}^{\mathrm{C}} \otimes \mathrm{T}_{2}^{\mathrm{V}} \text {. }
$$

With respect to $b^{*}$ the complete lift of the fundamental form $\tilde{\Omega}=\mathrm{d} \tilde{\eta} / 2$ is given by

$$
\tilde{\Omega}^{C}=\sum_{a}\left(d v^{a} \Lambda \omega^{a}{ }^{*}+w^{a} \Lambda d v^{a}{ }^{*}\right) .
$$

The exterior differentiation of (5.1) by means of (3.1) gives 


$$
\begin{aligned}
& \mathrm{d} \tilde{\Omega} \mathrm{c}=\breve{u} \wedge \tilde{\Omega}^{\mathrm{c}}+\sum_{a}\left(\breve{t}_{d} d \tilde{v}^{a^{*}}-\tilde{t}_{a}{ }^{*} d \tilde{v}^{a}\right) \wedge \tilde{\Omega}
\end{aligned}
$$

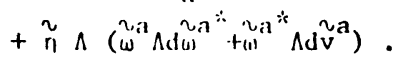

Using (5.2), we find

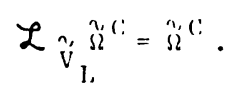

As is known (see Godbilion [21]), cqualion (5.3) shows that $\tilde{\Omega}^{\mathrm{C}}$ is homogeneous of degree 1 .

We will now take the complete lift $\tilde{u}^{\mathrm{C}}$ of the principal pfaffian $\tilde{u}$ associated with the c.c. connection with structures $\hat{M}$. For this purpose we shall denote by $\partial_{B}\left(\tilde{t}_{A}\right)=h_{B}\left(\tilde{t}_{A}^{*}\right)$ the Pfaffian derivatives of $\tilde{t}_{A}^{*} \quad(A=0,1, \ldots, 2 m)$ with respect to cobasis $\hat{W}^{*}$. Then according to the general theory (Yano and Ishihara [7]) one has

$$
\tilde{u}^{C}=\tilde{u}_{A} d v^{\sim A}+\partial_{B}\left(\tilde{u}_{A}\right) \tilde{v}_{\omega}^{B \sim A}
$$

where we have set $\tilde{u}=\tilde{u}_{A} \mathfrak{w}^{A}$. Referring to (3.4) and (3.5) (c=2), after some calculations one finds

$$
\begin{aligned}
& \tilde{u}^{C}=\frac{1}{2}\left[\left(\tilde{t}_{a}+\tilde{v}^{\mathrm{a}}-\tilde{t}_{a} d \hat{v}^{* a^{*}}\right)+\frac{1}{2}\left[\left(\tilde{t}_{a^{*}} \tilde{v}^{* a}-\tilde{t}_{a} \tilde{v}^{a^{*}}\right) \tilde{u}\right.\right.
\end{aligned}
$$

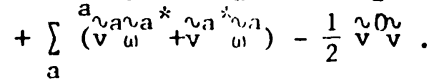

The exterior differentiation of (5.5) by means of (3.1) gives

$$
\begin{aligned}
& d \tilde{u}=\frac{1}{2}\left(\sum\left(\tilde{t} \tilde{v}^{a^{*}}+\tilde{t} a^{*} \tilde{v}^{a a}\right) \tilde{u}+\sum_{a}\left(\tilde{v} \tilde{v}_{w}^{a} a^{*}-v^{a^{*}} w_{w}^{a}\right)\right.
\end{aligned}
$$

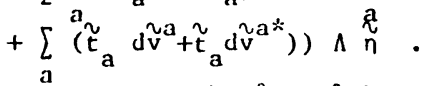
Using (5.5) and (5.6), one finds $\mathscr{L}_{v_{L}} \tilde{u}^{C}=\tilde{u}$. Hence $u^{C}$ is also a homogeneous form
of degree 1.

Consider now the following scalar field on $T \tilde{M}$ :

$$
\tilde{\tau}=\left[\tilde{v} \tilde{v}^{a^{*}}+\left(\tilde{v}^{0}\right)^{2} / 2\right.
$$

and apply the verticil differentiation of $\tilde{T}$. According to Godbillon [21], one has

$$
\tilde{v}=d_{v}{ }^{*}=\sum_{a}\left(\sim_{v}^{v a a^{n}, a^{*}}+\tilde{v}^{*}{ }^{*} \tilde{\omega}^{a}\right)+\tilde{v}_{n}^{0} \tilde{n}
$$

and by means of (3.1) one gets

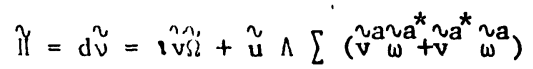

$$
\begin{aligned}
& +\tilde{n} \Lambda\left(\left[\left(v^{\sim a^{*}} \underset{\omega}{w a}-v^{a} a_{\omega}^{a} a^{*}\right)-d v^{0}\right)+2 \tilde{v} \tilde{\Omega}\right.
\end{aligned}
$$

In (5.9) $\quad:=\Lambda \tilde{M} \rightarrow C^{\infty} \eta \tilde{M}$ is the operator of Yano and lshihara [7], that is with respect to $B^{*}$ one has by (3.6)

One quickly finds

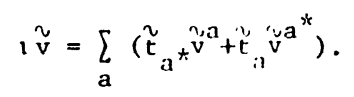

$$
\begin{gathered}
\qquad \mathfrak{v}_{L} \tilde{l}=\tilde{v}, \\
\text { and since } \pi \text { is closed, it follows from (5.11) that } \\
\mathcal{L}_{\vartheta_{L}} \tilde{l}=\tilde{H}
\end{gathered}
$$


1.e. If is homogeneous of degree 1. Noreover, taking the vertical derivation of II, one has (see Godbillon [21]):

$$
1_{v} \tilde{l}=0 \text {. }
$$

On the other hand, it is easy to see from (5.9) that II is of maximal rank (see (iodbilion [21]) on Tî. Accordingly, as is known, equations (5.11) and (5.13) prove that II is a Finsterian form (Sec r.l.in .ull voutier [22]). Since the vertical differentiation $d_{v}$ is an anti-derivation if square zero, one eastly dertves from (5.8) that

$$
\mathrm{d}_{\boldsymbol{v}} \tilde{v}=0, \quad i_{i_{l}} \ddot{v}=0 .
$$

Thus according to (iodbilton [21], $\tilde{v}$ is a smibasic form.

In the following we shall call $\hat{T}^{\prime}$ (resp. $v^{2}$ ) the liouvizze function (resp. the Liourvilile 1-form) on INî (see Rosca [16]). Further one may call il the $2-f(r) m$ of crretan on TM̈ (see Rosca [19]).

Denote now by $B=\left\{h_{A}, \frac{\partial}{\partial v \hat{v}}\right\}$ the vectorial basis dual to $B^{*}$ on $\tilde{M}$. Then as is known (see Yano and Ishihara [9] or (iodbillon [21]) the vertical 1ift $(\tilde{Z}) V$ of $\hat{V}$ is expressed by

$$
(\tilde{z})^{V}=\tilde{z} \frac{\partial}{\partial v^{A}}
$$

Coming back to the case under consideration and using that $U \tilde{U}=b^{-1}(\tilde{u})$ (see Section 3 ), we find by (5.15) that

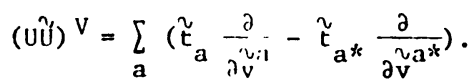

Now, taking the dual $\mu\left(u u^{\prime}\right)^{\prime}$ of (Uư) ${ }^{\prime}$ with respect to $\tilde{I}$ and referring to (3.5) $(c=2)$, we quickly find

$$
\mu(U \tilde{U})^{\prime}=2 \tilde{u} .
$$

Since $\tilde{u}$ and $\tilde{I}$ are both closed, it follows from this that $\mathscr{L}(U \tilde{U}) v^{\tilde{I}}=0$, $1 . e$. (UŨ) $v$ is an infinitesimal automorphism of $\tilde{\mathrm{ll}}$.

Consider now on IN the mechanical anstem $\partial \partial\{=\{\tilde{M}, \tilde{T}, \tilde{\pi}\}$ where $\tilde{T}$ and

$$
\tilde{u}=2 \cdot u
$$

are the kinetic energy and the field of jorres of $\partial 22$ (see Godbilion [21]). finds

Since $\tilde{u}$ is closed, one has $d \tilde{\pi}=\frac{d \tilde{T}}{\frac{T}{T}} \hat{n}$ and referring to (5.7), one quickly

$$
\begin{aligned}
& \vartheta_{L}^{\mu}=2 \dddot{T}, \\
& \vartheta_{L}{ }^{\pi}=2 \ddot{\prime} .
\end{aligned}
$$

Equations (5.19) show that $\tau$ and $\tilde{T}$ are hmognneous of degree 2 . On the other hand, since $\tilde{\pi}$ is an exact 2 -form of maximsl rank, it defines a potential sympletic structure on rî. Hence, according to the definition given by klein (see Godbillon [21]) the systein $\partial$ is regular.

Denote now by $\tilde{Z}_{\mathrm{d}}$ the dynamical systim assoctated with $\partial 2$. As is known, $\check{Z}_{\mathrm{d}}$ 
is defined via formula

Then:

$$
i_{z_{d}} \tilde{u}=d\left(\tilde{T}-\tilde{v}_{L} \frac{\tilde{T}}{L}\right)+\tilde{\pi} .
$$

a) Since $\widetilde{T}$ and $\tilde{\pi}$ are both homognicous and of the same degree, $\tilde{Z}_{d}$ is a spray on $\hat{\mathrm{H}}$, 1.e. $\left[\hat{V}_{\mathrm{L}}, \tilde{Z}_{\mathrm{d}}\right]=\tilde{Z}_{\mathrm{d}}$.

b) Since $\dddot{T}$ is of degree 2 , the 2 -form $\tilde{I}-(d \tilde{T}-\pi) \wedge$ dt $\varepsilon \Lambda^{2}($ T $\tilde{M} \times R)$ is an integral relation of invariance for $\tilde{Z}_{d}+\frac{\partial}{\partial t}$ (Lichnerowicz [5]).

THEOREM 3. Let Tî be the tangent bundle manifold having as a basis the manifold $\tilde{M}(U, \xi, \tilde{n}, \tilde{g})$ defined in Section 3 and let $\tilde{U}$ (resp. $\tilde{\Omega}$ ) be the principal vector field (resp. the fundamental 2-form) on $\tilde{M}$. Then:

(i) the complete 1 ifts $\tilde{\Omega}^{C}$ and $\tilde{u} \mathrm{C}$ of $\tilde{\Omega}$ and $\tilde{u}=b$ (UU) are homogeneous of degree one;

(ii) the 2-form of Cartan $\tilde{I}$ on TNं is a Finslerian form;

(iii) one may associate with $\hat{f}$ a regular mechanical system whose dynamical system is a spray on $\tilde{M}$.

\section{RFFERENCES}

1: RosCA, R. On Pseudo-Sasakian Manifolds, Rend. Mat. 1984 (to appear).

2. coldberc;, V.V. and ROSCA, R. Contact ('o-1sotroplc CR Submanifolds of a PseudoSasakian Manifold, Intern. J. Math. Math. Sci. I(1984), No. 2, 339-350.

3. ROSCA, R. Vartétés Sasakienne à Conmexion Conforme de Contact, C.R. Acad. Sci. Paris Sér. I Math. 294(1982), 43-46.

4. LICINEROWICZ, $A$. Les Relations Integrales d'Invariance et Leurs Applications à 1a Dynamique, Bul1. Sc1. Math. 70(1946), 82-95.

5. ROSCA, R. Variétés Lorentriennes à Structure Sasakienne et Admettant un Champ Vectoricl lsotrope N-quasi Concirculaire, C.R. Acad. Sci. Paris Ser. A. 291 (1980), 45-47.

6. LICHNERowICZ, A. Vartétés de Poisson et Feuilletages, Ann. Fac. Sc1. Toulouse Math (5) 4 (1982), 195-262.

7. YANO, K. and KON, M. CR Submanifolds of Kaehlerian and Sasakian Mantfolds, Birkhäuser, Boston-Basel-Stuttgart, 1983.

8. ROSCA, R. CR-sous-variétés Co-isotropes d'une Vartété Parakählerienne, C.R. Acad. Sc1 Paris Sér. I Math. 298(1984), 149-151.

9. YANO, K. and ISHIHARA, S. Differentia1 Geometry of Tangent and Cotangent Bundles, Marce1 Dekker Inc., New York, 1973.

10. KLEIN, I. Espaces Variationels et Mècanique, Ann. Inst. Fourier (Grenoble) $12(1962), 1-124$.

11. Libermann, P. Sur le Problème d'Équivalence de Certaines Structures Infinitésimales, Ann. Mat. Pura Appl. 36(1951), 27-120.

12. ROSC $\Lambda, R$. Codimenstion 2 CR Submanifolds with Null Covariant Decomposable Vertical Distribution of a Neutral Manifold, Rend. Mat. (4) 2 (1982), 787-796.

13. DATTA, D.K. Exterior Recurrent Forms on a Mantfold, Tensor (N.S.) 36 (1982), No. 1, 115-120.

14. POOR, W.A. Differential Geometric Structures, McGraw-H111 Book Comp., New York, 1981 .

15. ROSCA, R. and VERStraELEN, L. On Submanifolds Admitting a Normal Section Which is Quasi-concircular w.r.t. a Corresponding Principal Tangent Section, Bu11. Math. Soc. Math. R.S. Roumanie 20(68) (1976), No. 3-4, 399-402. 
16. ROSCA, R. On Parallel Conformal Comnertions, Kodai Math. J. 2(1979), No. 1, $1-10$.

17. SlNIIA, B.B. A Differentiable Manifold with Para f-Strurture of Rank $r$, Ann. Fac. Sci. Univ. Nat. Zaire (Klushasa) Sect. Math.-Phys. 6(1980), No. $1-2,79-94$.

18. Lichnerowicz, A. Feuilletages, Géométrie Riemannicnne et céométrie Symplectique, C.R. Acad. Sc1. Paris Ser. 1 Mitll. 296(1983), 205-210.

19. RUSCA, R. Espacc-temps Ayant la propriété liéndésique, C.R. Acad. Sci. Parls SÉr. A 285 (1977), 305-308.

20. ROSCA, R. Sous-variétés Anti-invariantes d'une Variété Parakahlertenne Structurée par une Connexton ('éodesique, C.R. Acad. Sc1. Paris Sér. $\Lambda$ 287(1978), 539-541.

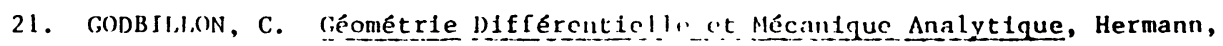
Paris, $196 \overline{9}$.

22. KLEIN, I. and vourIER, A. Formes exlérlouros généralrices de sprays, Anu. Inst. Fourier ((ircnoble) 18(1968), 241-268. 


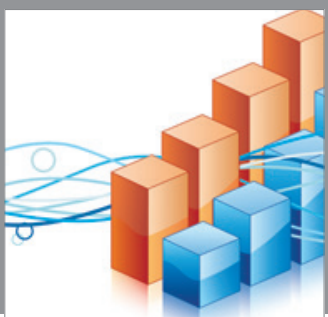

Advances in

Operations Research

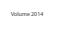

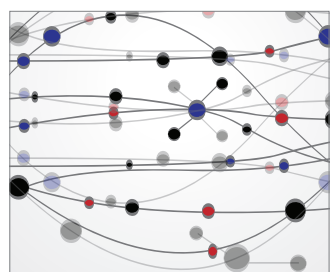

\section{The Scientific} World Journal
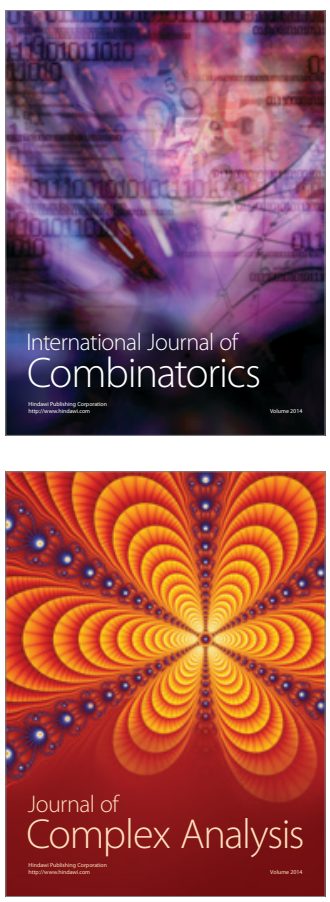

International Journal of

Mathematics and

Mathematical

Sciences
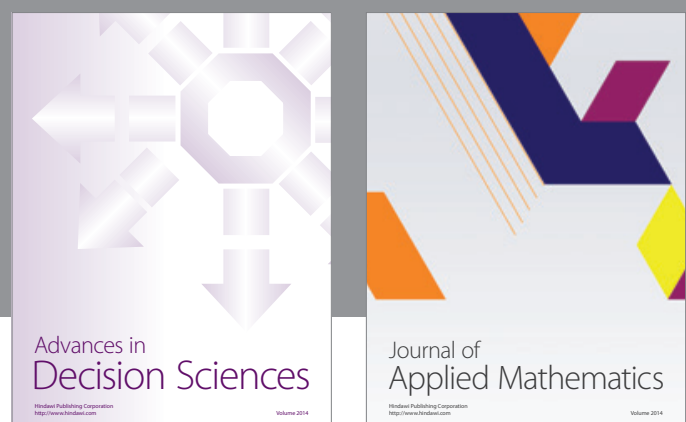

Journal of

Applied Mathematics
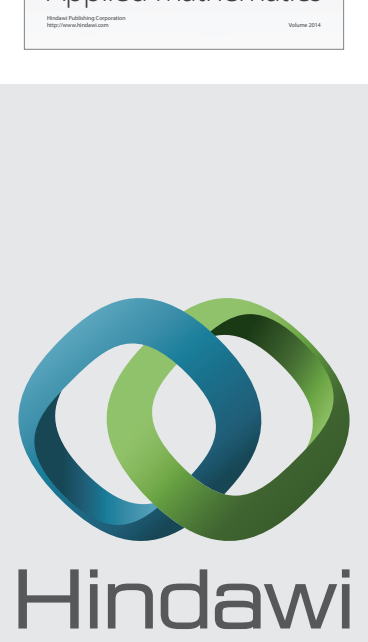

Submit your manuscripts at http://www.hindawi.com
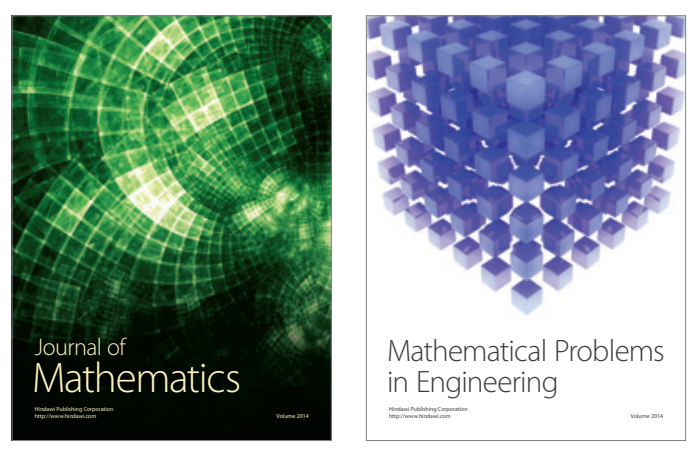

Mathematical Problems in Engineering
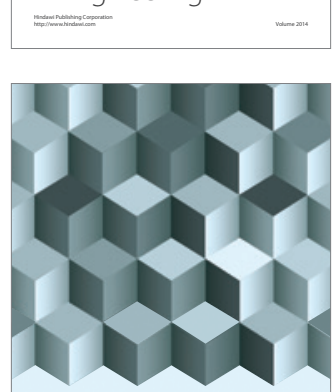

Journal of

Function Spaces
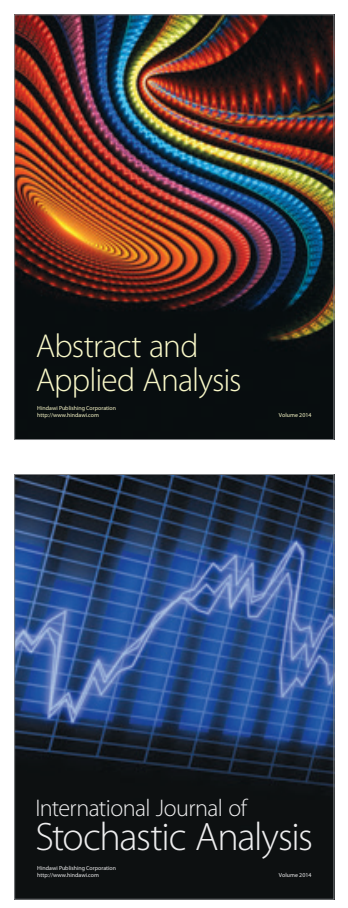

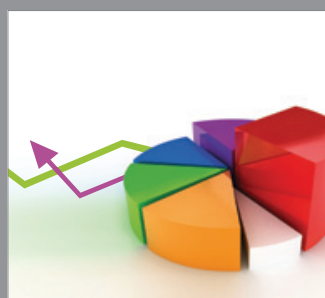

ournal of

Probability and Statistics

Promensencen
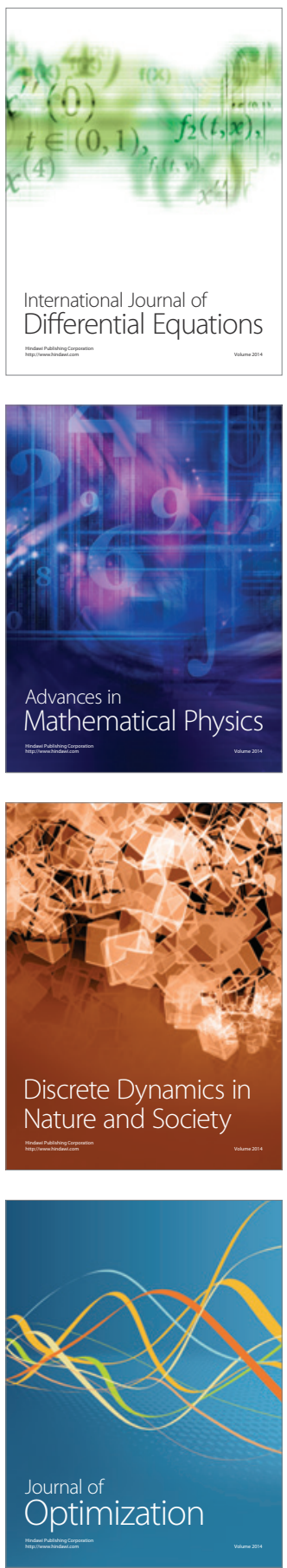